\title{
ALGUNOS ASPECTOS DE LA CUANTIFICACIÓN DE GRADO (CON ESPECIAL ATENCIÓN A LA LLAMADA "CUANTIFICACIÓN DE GRADO VERBAL”)*
}

\section{INTRODUCGIÓN}

Como señala en uno de sus trabajos I. Bosque, "Los avances realizados en el estudio de la llamada cuantificación sobre individuos han sido muy notables en los últimos años. Creo que en los próximos veremos algunos progresos en el análisis de la cuantificación sobre grados, es decir, sobre los niveles o los estadios en los que se aplican las propiedades o se evalúan los eventos" ${ }^{1}$. Desde que se publicó el artículo del que se citan estas palabras hasta la actualidad han salido a la luz algunos trabajos muy esclarecedores, sobre todo, en lo referente a la cuantificación de grado adjetival (aunque el punto de partida no sea exactamente sobre el tema, como veremos) y verbal ${ }^{2}$.

* La revisión de este trabajo, meses después de su envío a la Nueva Revista de Filología Hispánica, nos ha permitido consultar la Nueva gramática de la lengua española, Espasa Libros, Madrid, 2009, aunque la consulta refuerza la idea que en general preside este artículo: el análisis de trabajos muy particulares sobre el tema para seguir indagando en ciertos problemas relacionados con la cuantificación de grado, lo cual, en una obra de la envergadura de la que citamos garantiza generalizaciones necesarias, aunque prescinde de ciertos análisis muy específicos.

1 "Sobre la gramática de la gradación", Sociedad Chilena de Lingüística. Un aniversario especial, ed. A. Valencia, LOM Ediciones, Santiago de Chile, 2001, p. 40.

2 A. Vigueras Ávila, "Aspecto verbal y cuantificación adverbial gradual", VI Congreso de Lingüística Hispánica, Leipzig, 7 oct.-12 oct. 2003, trabajo inédito, pp. 1-16; I. BosQue, "Sobre la gramática...", pp. 39-79 y M.A. DALMAU, "Los verbos de locatum y la cuantificación verbal", RASAL. Lingüistica, 2004, núm. 1, 71-80, fundamentalmente. 
Pero la cuantificación de grado sigue presentando, en nuestra opinión, algunos interrogantes, tales como: $a$ ) ¿hasta qué punto está relacionada con la aspectualidad de ciertas unidades léxicas o bien es en mayor o menor medida independiente de ella? (Téngase en cuenta que la cuantificación de grado es entendida también como un caso particular de la delimitabilidad, que incluye la Aktionsart, modo de acción o aspecto léxico $\left.)^{3}, b\right)$ ¿se puede distinguir entre 'grado', 'cantidad', 'cualidad' e 'intensidad'?, y, sobre todo, $c$ ) ¿la cuantificación de grado, que es aceptada por distintas unidades léxicas, incide de la misma manera en todas ellas?, porque ¿qué pasa cuando el cuantificador de grado aparece, sobre todo, con un verbo: se trata en este caso de una auténtica cuantificación de grado verbal? D. Bolinger ${ }^{4}$ señalaba que las manifestaciones de grado e intensidad están asociadas comúnmente a adjetivos y adverbios, no a nombres y verbos. Sin embargo, también parecería frecuente la gradación, sobre todo, de eventos, aunque cuando decimos que "Alguien trabaja mucho", por ejemplo, no estamos reflejando un determinado grado de trabajar. Pues bien, a partir del análisis de algunas categorías que admiten cuantificadores de grado, podremos comprobar si se puede hablar propiamente de cuantificación de grado cuando este tipo de cuantificación no compromete propiedades, es decir, cuando lo que se gradúan son sustantivos y, sobre todo, verbos. Obsérvese que no estamos negando el hecho de que sustantivos y

${ }^{3}$ Cf. I. Bosque, "Sobre la gramática...", p. 64. No vamos a entrar en este trabajo en la polémica sobre la conveniencia o no de la terminología ni, lo que es más importante, si se tergiversó el concepto al pasar del estudio de las lenguas eslavas al de las lenguas románicas. Para responder a estas cuestiones están los estudios historiográficos de M. Fernández Pérez ("Sobre la distinción aspecto vs. Aktionsart”, Estudios de Lingüistica, 1993, 265-293), M. Rivas Zancarrón ("La aktionsart. Algunos intentos de adecuación al sistema verbal español", El verbo. Entre el léxico y la gramática, eds. A. Veiga, V.M. Longa y J.D. Anderson, Tris Tram, Lugo, 2000, pp. 167-175) y el estudio de J. Cuartero Otal ("Hacia otro modelo de análisis de la aspectualidad", Moenia, 2006, núm. 11, 197-228). Parece, en cambio, que en otros estudios en el ámbito hispánico se habla de aspecto léxico o aspectualidad sin que parezca un problema la diferente naturaleza morfológica del español (véanse, entre otros, E. DE Miguel, "El aspecto léxico", en Gramática descriptiva de la lengua española, dirs. I. Bosque y V. Demonte, Espasa Calpe-Real Academia Española, Madrid, 1999, t. 2, pp. 2977-3060; Y. MоRiмото, El aspecto léxico: delimitación, Arco/Libros, Madrid, 1998 y Los verbos de movimiento, Visor, Madrid, 2001).

4 Degree words, Mouton, The Hague-Paris, 1972, p. 15. 
verbos acepten cuantificadores de grado, sino que esos cuantificadores los afecten directamente, en particular, en el caso de los verbos.

No obstante, antes es necesario recordar que tanto en la cuantificación de grado como en la cuantificación sobre individuos u objetos intervienen un cuantificador y una variable; pero, además, como señala $\mathrm{H}$. López Palma ${ }^{5}$, en estas últimas aparecen, además del cuantificador y del término variable, la predicación restrictiva que expresa el rango de la variable, o la clase a la que pertenece, y el ámbito del operador, que es la función proposicional. Así, la lectura de "Todos los adolescentes son estudiantes", por ejemplo, sería la de "Para todo individuo $x$ tal que $x$ es un adolescente, $x$ es estudiante", donde "x es adolescente" es la predicación restrictiva (el rango de la variable x es el conjunto de los individuos "adolescentes"), y donde "x es estudiante" (proposición abierta) más el restrictor constituyen el ámbito en el que opera el cuantificador. Por tanto, la operación descrita se da cuando, según esta autora, "el cuantificador del lenguaje natural pertenece a la clase semántica de los operadores, e indica el número, la cantidad o la proporción de los objetos individuales que satisfacen el predicado. Su función característica como operador es ligar las variables de individuos que están dentro de su alcance..."6.

Ahora bien, en la cuantificación de grado el operador no liga variables relacionadas con los individuos u objetos que están dentro de su alcance, ya que la variable es de otra naturaleza, aunque esto haya provocado confusiones inexplicables con la cuantificación sobre individuos u objetos. Así, según C. Sánchez López, "cuantificar es expresar una cantidad, de ahí que los cuantificadores puedan definirse... como elementos que dicen qué cantidad de individuos u objetos de un dominio dado tienen una determinada propiedad, o en qué medida una propiedad es poseída por un individuo u objeto"7. Pero en el primer caso la cuantificación expresa cantidad, definida o no (se trata, pues, de una cuantificación sobre individuos u objetos), mien-

5 La interpretación de los cuantificadores. Aspectos sintácticos y semánticos, Visor, Madrid, 1999, pp. 27-44, libro en el que encontramos un más detallado análisis de los elementos que intervienen en las estructuras cuantificadas sobre individuos u objetos.

6 Ibid., pp. 44-45.

7 "Los cuantificadores: clases de cuantificadores y estructuras cuantificativas”, en Gramática descriptiva de la lengua española, t. 1, p. 1027. 
tras que en el segundo expresa el grado en que una propiedad se manifiesta (es una cuantificación sobre niveles, o cuantificación de grado). Esto puede ejemplificarse con la diferencia entre "muchos chicos faltaron a clase" y "la maestra se mostró muy contrariada".

Además, mientras que en la cuantificación sobre individuos u objetos el operador multiplica el valor de la variable, los cuantificadores de grado son operadores sobre los niveles en los que se aplican las propiedades, es decir, sus variables denotan niveles, no individuos u objetos. De ahí que algunos hablen estrictamente de cuantificación sólo en el primer caso; en cambio, C. Sánchez López (ibid., p. 1028) propone ampliar el inventario de elementos que permiten o exigen una lectura cuantitativa, entre los que se encuentran los cuantificadores de grado, lo que no justifica que incluya en esta última operación ejemplos como "Tiene más trabajo del que puede abarcar", u otros como "Ana tiene demasiados libros de sintaxis" y "Tiene muchos libros para ser un pésimo lector", que expresan pura "cardinalidad indefinida" (cf. ibid., pp. 1090-1091), es decir, cuantifican sobre objetos en estos casos.

\section{LOS CUANTIFICADORES DE GRADO}

\subsection{Tratamiento de los cuantificadores de grado en algunas gramáticas y en algunos trabajos específicos sobre el tema}

Antes de seguir con la idea de qué expresa la cuantificación de grado cuando se aplica a distintas categorías gramaticales, es la propia categoría gramatical del cuantificador de grado la que nos interesa determinar. En este sentido, la tradición gramatical ha incluido generalmente numerales, indefinidos, cuantitativos y, en este amplio marco, también cuantificadores de grado, en el mismo apartado, el de los pronombres que pueden funcionar como adjetivos ${ }^{8}$.

${ }^{8}$ Cf., entre otras, de S. Fernández Ramírez, Gramática española, 3.2: El pronombre, vol. preparado por J. Polo, Arco/Libros, Madrid, 1987 [1951]; Esbozo de una nueva gramática de la lengua española de la RAE, Espasa-Calpe, Madrid, 1982 [1973], y la Gramática española de J. Alcina y J. M. BlecuA, Ariel, Barcelona, 1975; no ocurre exactamente lo mismo en el Manual de gramática española, de R. SEco (Aguilar, Madrid, 1971). 
Pero el Esbozo de una nueva gramática de la lengua española añade un dato importante con vistas a nuestras consideraciones posteriores, y es el de que "por su capacidad de gradación cuantitativa, algunos de estos pronombres, en su forma neutra, actúan también como adverbios cuantitativos... Podemos decir que en estos casos y en otros más difíciles se produce neutralización o sincretismo entre las dos categorías" (p. 227). Los casos con los que ejemplifica el Esbozo son los de "Lloraba mucho" o "Reía poco", donde mucho y poco son adverbios; y los de "Ganó mucho" o "Perdió poco en el cambio", donde mucho y poco son pronombres cuantitativos neutros. Por último, en "Leía mucho", mucho, en cambio, pueden ser una cosa u otra, "según la intención del que habla (= Leía constantemente, adverbio; = Leía muchos libros, pronombre)".

En cuanto al Manual de gramática española, de R. Seco (p. 108), obra anterior al Esbozo, se trata de una de las pocas gramáticas que incluye mucho, poco, bastante, demasiado, apenas, casi, más, menos, etc., exclusivamente entre los "adverbios determinativos nominales" que expresan cantidad, y es esta adscripción la que nos interesa destacar.

En efecto, la cuantificación sobre individuos y objetos, por una parte, y sobre niveles, por otra, hacen uso de cuantificadores de naturaleza gramatical diferente, teniendo en cuenta también alguna bibliografía más reciente sobre el tema ${ }^{9}$. En concreto, los cuantificadores de grado pertenecen a la categoría adverbio (poco/bastante/muy alto, bastante/muy pronto, poco/bastante/muy hombre, trabajar poco/bastante/mucho), y son, por ello, invariables en cuanto a género y número.

Sólo un apunte en relación con ciertos adjetivos, denominados "adjetivos desnudos", equivalentes a cuantificadores de grado, hacen A. Suñer y A. Di Tullio ${ }^{10}$ en una ponencia presentada en el $11^{\circ}$ Coloquio de Gramática Generativa. Pero, si bien podría parecer que estos adjetivos equivalen a adverbios, las autoras no se refieren a casos como el de "Juan baila lento" (= de mane-

9 Véanse, en este sentido, C. SÁnchez López, art. cit., p. 1037 y, sobre todo, O. Kovacci, "El adverbio", en Gramática descriptiva de la lengua española, t. 1, p. 707.

10 "Los adjetivos desnudos y la cuantificación verbal", $11^{\circ}$ Coloquio de Gramática Generativa, Zaragoza, 4 de abril de 2001, pp. 1-12. Citamos la versión que se proporcionó a los alumnos asistentes al seminario titulado "Adjetivos: cuestiones morfológicas y sintácticas" (impartido en colaboración con L. Kornfeld) y celebrado en 2006 en la Facultad de Humanidades y Ciencias de la Educación de la Universidad de la República, Uruguay. 
ra lenta, frente a la otra posible interpretación: un baile lento), o a los de "Hablar claro" (= claramente) o "Trabajar duro" (= duramente), ya que, como trataremos de demostrar en el apartado 2.2, grado y manera no son equivalentes, sino a otros como "Este detergente lava blanco" (que no es equivalente a *lava blanquísimamente, pero sí a bastante blanco), o "Hiló fino" y "Cavó hondo". Y es que para que un "adjetivo desnudo" pueda desempeñar la función de un cuantificador de grado es necesario que ese adjetivo se refiera a un proceso o a un estado resultante (idea que desarrollaremos al hablar de los adjetivos perfectivos), no a personas u objetos, como sucede en "Él fuma rubio" (tabaco rubio), ni a manera referida al proceso, como ocurre en "Juan baila lento" (un baile lento), citado anteriormente.

Pero, en definitiva, lo que nos interesa es separar el estatuto de los cuantificadores de grado "habituales" respecto de otras categorías, sobre todo, para evitar confusiones del tipo de las que citamos más arriba, es decir, para lo que nos concierne en este trabajo, entre cuantificadores que expresan cardinalidad indefinida y cuantificadores de grado.

De todas formas, suele ser también de uso general en la lingüística contemporánea el término y el concepto de "cuantificador" en el sentido de categoría gramatical que expresa un número, una cantidad o un grado ${ }^{11}$. Esto implica reunir bajo una misma caracterización elementos adverbiales ("Te quiero mucho"), pronominales ("Hoy no he preparado mucho") o adjetivales ("Has tardado mucho tiempo"). Los que realizan esta adscripción no niegan la vinculación tradicional de los cuantificadores con las categorías de adverbio, pronombre y adjetivo, pero con ello establecen un gran número de generalizaciones sintácticas y semánticas. Preferimos, en cambio, la división entre numerales e indefinidos como subclases de los adjetivos y de los pronombres, por una parte, y adverbios de grado, por otra, aunque se pierda una generalización necesaria sobre esas palabras, pero se describa otra parte de su funcionamiento gramatical. Esta postura no impide que nuestra idea sea la de analizar el comportamiento semántico de los adverbios de grado y su incidencia en las unidades a las que acompañan, como trataremos de hacer a continuación.

11 Véase, de nuevo, C. SÁnchez López, art. cit., pp. 1025-1128 y H. López Palma, op. cit. 


\subsection{Distinción entre 'grado', 'cantidad', 'cualidad' e 'intensidad'}

Desde un punto de vista, a nuestro entender, estrictamente semántico, para C. Sánchez López los cuantificadores gradativos "se caracterizan por expresar el grado de cantidad, número o intensidad con que se toma una determinada realidad, esto es, por expresar una cantidad relativa respecto de algún parámetro que funciona a modo de escala"12. La idea suscita la pregunta de si hay, sobre todo, un grado de cantidad (a los efectos que nos ocupa, el número es también cantidad) y un grado de intensidad, o el grado es una noción diferente a la cantidad, a la intensidad y, añadiremos, a la cualidad.

Respecto al "grado de cantidad", en primer lugar, no queda claro en el planteamiento de C. Sánchez López si este "grado de cantidad" es distinto de la cantidad expresada por la cuantificación de individuos u objetos. En un determinado momento parece dar a entender que sí es diferente, y que el primero podría corresponder a la cuantificación de nombres continuos (much a o poca agua), pues señala que esta cuantificación difiere de la de los no continuos en que no expresa cardinalidad sino mera cantidad (además, dice que "los cuantificadores que pueden modificar nombres continuos son los que hemos incluido en el subgrupo de los indefinidos de grado"13).

En nuestra opinión, todos estos ejemplos, tanto de cuantificación de nombres discontinuos como continuos, formarían parte de la cuantificación de individuos u objetos (indicando número o simple cantidad), mientras que la cuantificación de grado no opera nunca sobre individuos u objetos.

Pero además está el tema de si el grado lleva implícita la noción de cantidad, o más bien la de cualidad. Así, parece que hay diferencias semánticas evidentes entre muchos libros, mucho trabajo o, incluso, much a paciencia ("cardinalidad indefinida" en estos casos), por una parte, muy alto o muy hombre (cuantificación de grado), por otra, e, incluso, finalmente, trabajar mucho (que puede indicar "durante mucho tiempo" o "que tiene mucha cantidad de trabajo"). Y, por tanto, parece que cuando hay cuantificación de grado la noción expresada, al menos en los casos citados de muy alto y muy hombre, y otros muchos, es no tanto cuantitativa como cualitativa.

12 Art. cit., p. 1031. Cf. también la p. 1090.

13 Op. cit., pp. 1057-1058. 
Sin embargo, las pruebas de comportamiento sintáctico no arrojan demasiada luz al respecto, aunque si bien los tres primeros ejemplos responden claramente a un cuánto (denominado "interrogativo de cantidad y grado"; en mi interpretación, sólo interrogativo de cantidad): "¿cuántos libros, cuánto trabajo o cuánta paciencia tiene?”; los del segundo grupo parecerían responder más bien a un cómo ("interrogativo de modo", al que responden tanto el modo como el grado), de manera que a la pregunta ¿cómo es? se responde en general "muy alto" o "muy hombre". Esto último no se cumple en el caso de "trabajar mucho", que sólo admite ¿̨cuánto trabaja? Por otra parte, la propia indistinción en una gran cantidad de gramáticas entre adverbios de grado y de cantidad contribuye a la dificultad de responder a la pregunta de si grado y cantidad son nociones distintas o no.

De la misma manera, hay que reconocer que la gradación se ha identificado muchas veces con la intensidad ${ }^{14}$. En este sentido, D. Bolinger ${ }^{15}$ dice que usa el término intensificar para hacer referencia a cualquier recurso que determina el nivel de una cualidad dentro de una escala, hacia arriba o hacia abajo o en algún lugar entre los dos extremos de dicha escala.

En un trabajo anterior, I. Bosque ${ }^{16}$ habla de los llamados "intensificadores" como muy y mucho, en casos como "un coche muy lleno" o "una persona muy enferma" 17 , frente a los "cuantificadores distributivos", en casos como "un autor muy conocido". Sin embargo, posteriormente argumentará que no es posible trasladar los adverbios de grado al grupo de los de manera, con lo cual el grado debe distinguirse de la intensidad ${ }^{18}$.

Hasta ahora se ha comentado que los cuantificadores de grado pertenecen a la categoría adverbio, lo cual es importante recordar habida cuenta del diferente tratamiento de estas unidades en distintas gramáticas (cf. 2.1). Pues bien, independientemente de que I. Bosque exponga esta distinción entre grado

${ }^{14}$ Si bien en la Gramática española de J. Alcina Franch y J. M. Blecua (Ariel, Barcelona, 1989) hay un apartado dedicado a los cuantitativos gradativos y otro a los intensivos, la distinción no queda clara en esos apartados ni en otras partes de esta gramática (véanse, sobre todo, las pp. 573, 636 y 652).

15 Op. cit., p. 17.

16 "Sobre el aspecto en los adjetivos y en los participios", Tiempo y aspecto en español, ed. I. Bosque, Cátedra, Madrid, 1990, p. 194.

17 Véanse también V. Demonte, "El adjetivo: clases y usos. La posición del adjetivo en el sintagma nominal”, en Gramática descriptiva de la lengua española, t. 1, p. 173 y O. Kovacci, art. cit., pp. 713 y 779.

18 "Sobre la gramática de la gradación", pp. 61-63. 
e intensidad a partir del análisis de la cuantificación de grado verbal, de la que hablaremos más adelante, lo importante de su desarrollo en este momento reside precisamente en plantear la diferencia, que se apoya en el hecho de que los adverbios de intensidad presentan restricciones que no tienen los de grado. En este sentido, mientras que poderosamente, por ejemplo, se usa sólo con influir, atraer y llamar la atención, no podemos hacer listas de "idiosincrasias léxicas" para prever lo que sucede, por ejemplo, en "ensanchar más la sala", "llover mucho", "ensuciar demasiado la ropa", etc. ${ }^{19}$.

Pero, además, es necesario aludir, desde el punto de vista léxico, al contenido de 'fuerza' (y no al de 'en un grado elevado') que presenta la noción de intensidad ${ }^{20}$, idea de 'fuerza' que no parece constatable en ejemplos como "muy alto", "muy tarde", "muy hombre" o "trabajar mucho", aunque parece que sí en "gritar mucho", "querer mucho", "recordar mucho" o "masticar mucho". No obstante, respecto a la llamada "cuantificación de grado verbal", la cuestión no parece estar en decidir entre grado e intensidad, por cuanto de particular tiene la "gradación" de eventos, como veremos en 4 .

\section{CATEgorías GRAMATICAles QUE ACEPTAN CUANTIFICADORES DE GRADO}

A continuación vamos a hacer una descripción de las categorías que aceptan cuantificadores de grado, con excepción de los adverbios, ya que creemos que está claramente descrita la naturaleza aspectual de los adverbios que admiten y que se ven afectados por los cuantificadores de grado; al menos no parece que existan grandes inconvenientes en vincular la naturaleza aspectual de esta categoría con la posibilidad de aceptar tal tipo de cuantificadores. Brevemente, entre los adverbios es necesario distinguir entre los que denotan una zona acotada o un punto preciso en el espacio (debajo, encima, detrás, delante), que serían delimitados, y que no pueden graduarse, y los que representan una situación espacial aproximada o sin límite inherente, los no delimitados, que admitirían, por tanto, gradación (abajo, arriba, adelante, atrás, cerca, lejos); de esta manera, aceptaríamos muy

19 Cf. ibid., pp. 61-62.

20 Agradecemos a I. Bosque la indicación. 
abajo pero no *muy debajo, o muy atrás pero no *muy detrás, etc. Igualmente se puede distinguir, desde la perspectiva temporal, entre los adverbios que representan una zona acotada (ayer, mañana), que no pueden graduarse, y los que representan conceptos graduales, al indicar una zona sin límite (pronto y muy pronto, tarde y muy tarde) ${ }^{21}$.

En cambio, no es tan fácil establecer una tipología de los adjetivos que admiten cuantificadores de grado, como se observa en estudios muy exhaustivos sobre esta categoría que aluden también a su posibilidad de graduarse ${ }^{22}$; en efecto, tales estudios demuestran que si bien existe una amplia gama de adjetivos que aceptan este tipo de cuantificadores, otros no, y las subclases de estos adjetivos se entrecruzan.

En cuanto a los sustantivos, en ellos se dan unas circunstancias especiales, porque aunque aparentemente se podría anteponer un cuantificador de grado a cualquier sustantivo ("?esta mesa es mucha mesa"), normalmente la operación de cuantificación de grado necesita "conciliarse" con una serie de propiedades que también estén presentes en algunos sustantivos.

$Y$, finalmente, en el caso de los verbos, el hecho de que acepten cuantificadores de grado no quiere decir, ni mucho menos, que tales cuantificadores incidan directamente sobre ellos. Así, cuando un cuantificador de grado acompaña a un verbo nos encontramos con distintas interpretaciones semánticas que hacen incluso que nos cuestionemos la existencia de una auténtica cuantificación de grado verbal en todas ellas. Es más, una de las interpretaciones semánticas que consideraremos en 4.2, la denominada "interpretación inherente", incide justamente en el hecho de que la llamada "cuantificación de grado verbal" constituye, como señala I. Bosque, una paradoja, ya que "los eventos permiten modificadores de grado, pero no aportan ninguna variable sobre los niveles (extents) en los que se predican" 23 .

${ }^{21}$ Los ejemplos están extraídos del trabajo de Y. Morimoto, El aspecto léxico, pp. 33-34.

22 Véase, sobre todo, el esclarecedor trabajo de V. Demonte (art. cit., pp. 129-215).

${ }^{23}$ Cf. I. Bosque, "Sobre la gramática de la gradación”, p. 67. 


\subsection{Clases de adjetivos que admiten gradación}

Tanto en lo que llevamos expuesto a lo largo de este trabajo como en otro anterior ${ }^{24}$ hemos hecho notar la relación existente entre la cuantificación de grado y la aspectualidad. Pero ya I. Bosque ${ }^{25}$ había señalado que si bien la determinación de clases aspectuales es muy importante para comprobar las restricciones léxicas que estas clases son susceptibles de producir (así, el aspecto léxico nos permite entender un gran número de alternancias entre predicados y una parte de su estructura argumental, la formación de perífrasis verbales o algunas de las propiedades de los adverbios de tiempo y aspecto, entre otras cuestiones), tales clases aspectuales no son suficientes para determinar todas las restricciones léxicas posibles.

Trasladando esta constatación a la relación entre los predicados $^{26}$ delimitados y no delimitados ${ }^{27}$ y la cuantificación de grado adjetival, creemos que no han de establecerse, al menos, generalizaciones simplistas en el sentido que exponemos a continuación.

Así, en términos generales puede seguir manteniéndose el hecho de que se gradúan, según los ejemplos de algunos auto$\mathrm{res}^{28}$, los adjetivos calificativos ("biblioteca muy amplia", "discurso más solemne", "control demasiado severo"), pero no

${ }^{24}$ Cf. M.D. MuÑoz NúÑEz, "En torno al concepto de gradación o cuantificación de grado", en Actas del VI Congreso de Lingüistica General (Santiago de Compostela, 3-7/05/2004), Arco/Libros, Madrid, 2006, pp. 2195-2205.

25 "Bases para un diccionario de restricciones léxicas", Moenia, 2001, núm. 7, p. 13.

${ }^{26}$ Empleamos el término predicado, como es habitual en la bibliografía actual, para aludir a todos aquellos elementos que seleccionan sus argumentos y que, al hacerlo, restringen el tipo de unidades con las que pueden combinarse en función de determinados rasgos semánticos (véanse I. BosQUE, "Bases para un diccionario...", pp. 11-52 y "Sobre el concepto de colocación y sus límites", LEA, 23, 2001, 9-40, y C. Subirats RÜGGEBERG, Introducción a la sintaxis léxica del español, Vervuert-Iberoamericana, Frankfurt/M.-Madrid, 2001, pp. 201 ss.).

${ }_{27}$ Se trata de una distinción básica reconocida desde la Antigüedad bajo denominaciones como la de verbos de kinesis y de energeia, empleadas por Aristóteles, o verbos desinentes -con término-y permanentes-sin término-, empleadas por A. Bello (Gramática de la lengua castellana, Edaf Universitaria, Madrid, 1980 [1847], p. 200), y posteriormente con denominaciones como las de resultativos-no resultativos, perfectivos-imperfectivos, télicos-atélicos, etc. (véase, entre otros, E. DE Miguel, art. cit., p. 3019, en especial la n. 54).

${ }^{28}$ En concreto, estos ejemplos están extraídos también del trabajo de Y. Morimoto (El aspecto léxico, p. 35). 
los relacionales (“*biblioteca muy municipal”, “*discurso más papal", "*control demasiado aduanero"), ya que los primeros representan cualidades relativas -por tanto, pueden situarse en distintos puntos de una escala que admite diversos grados-, mientras que los relacionales "no denotan cualidades o propiedades de los sustantivos sino que... establecen conexiones entre esas entidades y otros dominios o ámbitos externos a ellos" ${ }^{29}$.

Pero si atendemos las exhaustivas clasificaciones sobre los adjetivos que se han hecho ${ }^{30}$, la relación entre adjetivos calificativos y 'no delimitación', por una parte, y adjetivos relacionales y 'delimitación', por otra, puede resultar, en efecto, demasiado simplista atendiendo a que distintos grupos de adjetivos que presentan un carácter más bien próximo a la delimitación, o que son claramente delimitados, son susceptibles de graduarse ("muy lleno", "bastante limpio"), de la misma manera que algunos calificativos, aparentemente con carácter no delimitado, no se gradúan ("actuación constitucional”, pero no "*actuación muy constitucional"). Por tanto, habría que determinar clases más concretas de adjetivos que acepten gradación. Además, en principio al menos, no parece tener demasiado sentido poner en correspondencia los adjetivos relacionales con la 'delimitación’ y, por tanto, con la no gradación, ya que estos adjetivos no expresan propiamente cualidades ${ }^{31}$.

Si analizamos algunos trabajos específicos sobre la categoría adjetivo, resulta muy interesante, fundamentalmente, el ya mencionado de V. Demonte ${ }^{32}$, que, aunque no está dedicado exclusivamente a la propiedad que tienen algunos adjetivos de graduarse, sí alude en distintos momentos a esta circunstancia. Así, cuando hace referencia a las clases de adjetivos según las relaciones semánticas que contraen con los nombres ${ }^{33}$, es de destacar, por ejemplo, la existencia de adjetivos calificativos individuales, que se predican con el verbo ser, que no aceptarían

29 I. BosQue, "Sobre las diferencias entre los adjetivos relaciones y los calificativos”, Revista Argentina de Lingüistica, 1993, núm. 9, p. 10.

${ }^{30}$ Véanse, sobre todo, I. BosQue, "Sobre el aspecto en los adjetivos...", pp. 177-214 y "Sobre las diferencias entre los adjetivos...", pp. 9-48, y V. Demonte, art. cit., pp. 130-215.

31 Agradecemos la sugerencia a S. Costa, profesora de la Universidad de la República, Montevideo, ya que consideramos que el comprometer propiedades es esencial en nuestra propuesta de aceptación de cuantificación de grado en lo que se refiere a los adjetivos, como expondremos más adelante.

32 Art. cit., pp. 129-215.

33 Ibid., pp. 141-149. 
cuantificadores de grado (“*muy psicópata”, “*muy idóneo”), y de calificativos absolutos que tampoco se graduarían ("*muy cuadrado", "*muy redondo"), entre otras subdistinciones dentro de los adjetivos calificativos.

En la línea de que ciertos adjetivos acepten la predicación con ser o estar, es de destacar también el comportamiento de los adjetivos perfectivos, que se predican con estar, y que necesitan de un argumento eventivo, es decir, un argumento que indica estado resultante ${ }^{34}$, algunos de los cuales, sin embargo, se gradúan. Tomando, pues, como punto de referencia la predicación con ser o estar, podemos concluir que ciertos adjetivos calificativos que se combinan con ser no se graduarían, y ciertos otros, en este caso perfectivos, que se combinan con estar, sí se graduarían (como los casos citados de "muy lleno", "bastante limpio" o el de "muy enfermo"), con excepción de otros casos de adjetivos perfectivos que se combinan con completamente, donde este adverbio no es de grado sino de aspecto ("completamente desnudo", "completamente vacío", frente a "*completamente magnífico"), como bien señala I. Bosque ${ }^{35}$.

Pero todo esto sólo indicaría, en nuestra opinión, la tendencia de adjetivos calificativos, en sus distintas subclases, y perfectivos a expresar en mayor o menor medida propiedades (lo que se da también en algunos casos de adjetivos relacionales que tienen una lectura calificativa: "un estilo muy inglés", "un coche muy económico"), que es el ámbito donde creemos que tiene sentido la cuantificación de grado.

\subsection{Algunos sustantivos susceptibles de graduarse}

Como decíamos en 1, D. Bolinger ${ }^{36}$ señalaba que las manifestaciones de grado e intensidad están asociadas comúnmente a adjetivos (como, en español, "muy alto") y adverbios ("muy lentamente"), no a nombres y verbos, aunque también es frecuente la "gradación" de estas otras categorías ("muy torero", "muy hombre", "muy mujer", "*muy mesa/mucha mesa"; "trabajar mucho", "recordar mucho", "querer mucho", "gritar mucho").

${ }^{34}$ Cf., para ello, C. SÁnchez López, art. cit., pp. 3019-3020, y, sobre todo, I. Bosque, "Sobre el aspecto en los adjetivos...," pp. 177-214.

35 "Sobre el aspecto en los adjetivos...", p. 193.

36 Op. cit., p. 15. 
En este punto, consideramos que el análisis algo más detenido de otras categorías susceptibles de graduarse, como son ahora el sustantivo y el verbo, podría arrojar más datos respecto al comportamiento de la cuantificación de grado, sobre todo respecto a si no se está utilizando el concepto de cuantificación de grado para aludir simplemente a la idea de ordenar en una escala, lo que no la haría diferente a la cuantificación de individuos u objetos, cuando en realidad debe pensarse que es distinta, tanto en los casos de cuantificación de grado adjetiva, sustantiva y, sobre todo, en el caso de la llamada "cuantificación de grado verbal", diferente en líneas generales también de la adjetiva y sustantiva.

En efecto, tanto la cuantificación de individuos u objetos como la de grado son escalares. Respecto al primer tipo, $\mathrm{H}$. López Palma, basándose en la opinión de R. Rivara, señala que tanto la cuantificación numérica como la denominada por esta autora "valorativa" (muchos, pocos, bastantes, demasiados, etc.) operan "sobre una escala dimensional y se establecen mediciones o se calculan cantidades tomando como base la escala dimensional, que es una escala numérica en la cuantificación cardinal y una escala de cantidades en la cuantificación valorativa" ${ }^{37}$. Insistimos, en cambio, en que la diferencia entre la cuantificación de individuos u objetos y la de grado podría residir en que la segunda es más bien cualitativa, al menos en lo que se refiere a su aplicación a muchos adjetivos y algunos sustantivos, aunque no parece que podamos hacer las mismas consideraciones respecto al verbo.

Pero detengámonos un poco más en lo que ocurre con los sustantivos que admiten gradación, porque en principio los nombres no pueden graduarse por el hecho "de involucrar un conjunto de criterios y dificultar así el que se sepa qué rasgo sería el que se está comparando o midiendo" 38 .

Conviene señalar que frente a la postura que considera que tales sustantivos se han recategorizado como adjetivos ${ }^{39} \mathrm{y}$, por tanto, pueden graduarse, está la de los que consideran que la

37 Op. cit., p. 50, n. 40.

38 V. Demonte, art. cit., p. 139.

39 Es interesante destacar que en un trabajo reciente de A. Di Tullio y L. KORNFELD ("Condiciones para la conversión de nombres a adjetivos en español”, III Encuentro de Gramática Generativa, 18-20 de agosto de 2005, 2005, p. 6) se aluda al hecho de que el rasgo 'humano' intervenga en esta "conversión categorial", argumentando para ello que no se trata meramente de 
gradación no es un criterio de identificación categorial, por lo que no podemos afirmar que una determinada palabra pertenece a una categoría por el hecho de admitir gradación; más bien que la gradación concierne a la semántica de una determinada unidad, no a su categoría. En este sentido, de la misma manera que algunos adjetivos relacionales se pueden graduar y pasar a calificativos (“un estilo muy inglés"), en el caso de sustantivos que se pueden graduar no estamos ante un proceso de adjetivación, sino que "la lengua establece para algunos sustantivos a los que históricamente se asocian significados culturales estereotipados la posibilidad de pasar a formar parte de las unidades léxicas que denotan cualidades o propiedades" ${ }^{\prime 4}$. Unido a esto, resulta también interesante la apreciación de I. Bosque de que es necesario descartar de antemano las estructuras sintácticas en las que prácticamente cualquier sustantivo se puede graduar: "por muy presidente del gobierno que sea", ya que aquí el adverbio de grado pertenece a una pauta sintáctica que no permite deducir ninguna propiedad de los sustantivos que entran en ella.

Pero habría que pensar que si bien es fácil aceptar que en el caso de "muy torero" estamos ante una unidad sustantiva que denota cualidad, no aceptaríamos que las cosas sean así en "*muy mesa" (aunque quizá sí en "mucha mesa" -"Esta mesa es mucha mesa"-), porque no estamos ante un significado cultural estereotipado. Sin embargo, las categorías gramaticales que se están combinando y el significado son los mismos en ambos casos. Obsérvese, por lo demás, la posible alternancia entre muy y mucho en casos como: "muy/mucha madre", "muy/ mucha mujer", "muy/mucho hombre", etc., aunque quizá no en "muy/?mucho torero", y los escasos ejemplos sólo con mucho como cuantificador de grado, y no como cuantificador indefinido, que se combina con el sustantivo en casos como "mucha mesa". Es de suponer que la elección entre muy y mucho según el sustantivo de que se trata se debe a una cuestión de norma, aunque la interpretación sea más bien cualitativa, como en los $\operatorname{adjetivos}^{41}$.

una propiedad léxico-semántica sino de un rasgo formal gramaticalmente relevante que interviene en la sintaxis (ibid., p. 10).

${ }^{40}$ I. BosQue, Las categorías gramaticales. Relaciones y diferencias, Síntesis, Madrid, 1989, p. 124.

${ }^{41}$ Pero no descartamos que haya un combinado de cualidad y cantidad en todos estos casos de cuantificación de grado adjetiva y sustantiva. 
Por lo demás, hay que insistir, en nuestra opinión, en que ésta es la única cuantificación de grado que puede admitir el sustantivo, y no la operación que cuantifica individuos u objetos (es decir, que en realidad indica cantidad), como sucede en "muchos problemas", "mucho trabajo" o "mucha paciencia", y que ha sido descrita a veces, junto con los casos desemejantes como "bastante alto", "poco/un poco torpe", "muy tarde", etc., como cuantificación de grado sobre una escala cuantitativa ${ }^{42}$.

\section{El PROBLEMA DE LA "CUANTIFICACión DE GRAdo VERBAL"}

En una contribución presentada al VI Congreso de Lingüistica General (Santiago de Compostela, 3-7/05/2004) ${ }^{43}$ expusimos brevemente la problemática de este tipo de cuantificación, partiendo de la base de que, como bien señaló I. Bosque ${ }^{44}$, el hecho de que los modificadores de grado cuantifiquen sobre los estadios en que tienen lugar los eventos no significa nada, ya que en realidad los verbos no aportan ningún tipo de variable de grado, es decir, ningún tipo de variable sobre los niveles en los que se predican.

Desde la publicación del trabajo de I. Bosque, anteriormente citado, hasta la actualidad no han sido muchos los estudios que han ahondado en este tema, si bien la bibliografía sobre aspecto léxico y cuantificación en general es hoy en día prácticamente inabarcable. Pero, considerando la posibilidad que ofrecen muchos verbos de aceptar cuantificación de grado (otra cuestión será la de la verdadera incidencia en el verbo del cuantificador), el panorama se reduce considerablemente.

A continuación trataremos de ofrecer un desarrollo sobre el tema, insistiendo precisamente en la idea de si en realidad estamos ante una paradoja de la cuantificación de grado, porque el cuantificador no tenga como variable un verbo, o si se acepta la idea de la existencia de un cuantificador de grado que incide de alguna manera sobre éste. Como hace entender I. Bosque (p. 68), desde el punto de vista sintáctico el cuantificador de grado es un adjunto o circunstante que modifica al verbo.

42 Cf. C. Sánchez López, art. cit., pp. 1098-1100.

43 Cit. supra, n. 24.

44 “Sobre la gramática de la gradación”, p. 67. 


\subsection{La "cuantificación de grado verbal" desde la óptica de algunas clasificaciones aspectuales}

En la línea de aceptación de que el cuantificador de grado tenga como variable un verbo, C. Sánchez López señala que el criterio que determina tal admisión no es categorial sino semántico, es decir, su significado se debe adecuar a ciertos requisitos. Así, según la autora ${ }^{45}$, hay restricciones semánticas que limitan el hecho de que los verbos o los SSVV admitan cuantificadores de grado. Pero no sólo habla de tales restricciones en casos como los de "Juan nada mucho", “Por qué dices que Juan habla mucho de política?", etc., sino que en ejemplos como los de "Juan grita mucho cuando habla" o "Juan te quiere mucho" pone de manifiesto, además, que el "cuantificador tiene una interpretación que corresponde a una verdadera cuantificación de grado" (id.): tanto gritar como querer denotan acciones que pueden hacerse en distinto grado sin que supongan acciones diferentes; en cambio, nadary hablar denotan acciones absolutas, no graduables. Y concluye con la idea de que aunque muchos verbos admiten un cuantificador como mucho, el significado de éste será distinto: "quejarse mucho" puede ser "quejarse intensamente", pero "dormir mucho" no es "dormir profundamente" sino "durante mucho tiempo".

Precisamente en nuestro trabajo citado antes ${ }^{46}$ señalábamos que si las interpretaciones son distintas a las de grado, la cuantificación no tiene, pues, tal carácter y el cuantificador no es de grado; así, en el caso de "dormir mucho", el cuantificador tiene como variable un sustantivo continuo, tiempo, y no difiere por eso de la cuantificación que indica cardinalidad indefinida (cf. apartado 1 y, también, el 4.2).

Pero antes de analizar más detenidamente esas falsas interpretaciones de grado es necesario hacer referencia a otro trabajo que se perfila en una línea parecida al de C. Sánchez López, es decir, que no cuestiona exactamente el hecho de que el cuantificador de grado incida realmente en el verbo, pero que tiene la particularidad de hacer un exhaustivo análisis sobre la naturaleza aspectual de los verbos que aceptan cuantificadores de grado. Nos referimos al trabajo inédito de

\footnotetext{
45 Art. cit., p. 1094.

${ }^{46}$ Art. cit., p. 2203.
} 
A. Vigueras Ávila, "Aspecto verbal y cuantificación adverbial gradual" 47 .

Partiendo de una clasificación aspectual de los verbos básica en la literatura sobre el tema ${ }^{48}$, la que establece la distinción entre estados, actividades, efectuaciones y logros, A. Vigueras ${ }^{49}$ argumenta sobre por qué los verbos de actividad son los que más aceptan los cuantificadores de grado; en segundo lugar, los verbos de estado; les siguen los de logro, siendo las efectuaciones las únicas que no los admiten.

Así, no tenemos inconveniente alguno en aceptar las siguientes actividades acompañadas de un cuantificador de grado, aportando informaciones como las que se citan a continuación: "Jorge nada mucho" (= muchas veces, media hora, diez kilómetros), "Come mucho" (= mucha comida, a cada rato) o "Llora mucho" (= muy a menudo); en estos casos, los correspondientes verbos aceptan el cuantificador de grado porque las actividades son expandibles en un dominio temporal y espacial. También son aceptables las expresiones con verbos de estado como "Sara se parece mucho a su abuela" y "Ella cree mucho en Dios", aunque no "Los demonios existen mucho" ni "Ella cree mucho que tiene un virus"; y es que, según A. Vigueras Ávila (cf. ibid., p. 10), el único dominio expandible en los estados es el dominio mental de un participante de la predicación, que en ocasiones concuerda con el sujeto, lo cual no es posible en los dos últimos casos. Por otra parte, son igualmente posibles expresiones con verbos de logro como "Los niños se caen mucho cuando aprenden a caminar" y "Se despierta mucho cuando tiene pesadillas", pero no "Antonia muere mucho"; en estos casos sucede que el único dominio que se puede expandir, al ser la acción puntual, es el iterativo. Finalmente, no es posible, en cambio, ninguna expresión con verbos de efectuación, como ocurre en "Ella escribe mucho un poema" o "Juan construye mucho la casa".

47 Un resumen de este trabajo apareció en el libro de resúmenes del VI Congreso de Lingüistica Hispánica, Leipzig, 7 oct.-12 oct. 2003. E1 trabajo completo no fue publicado, por lo que agradecemos a su autora que nos lo haya proporcionado.

${ }^{48}$ Que se remonta a Z. Vendler, "Verbs and times", The Philosophical Review, 66 (1957), 143-160.

49 Art. cit., p. 2. 
4.2. Distintas interpretaciones de la "cuantificación de grado verbal" más allá de las clasificaciones aspectuales básicas

Siguiendo con el análisis concerniente a la supuesta gradación de eventos, hemos adelantado la postura de I. Bosque, que sería la que corresponde al hecho de que la verdadera variable del cuantificador que acompaña al verbo no es ésta.

Sin embargo, en una parte de su argumentación acepta que en algunas expresiones nos podemos encontrar con varias interpretaciones, sin aludir a cuál sería la variable correspondiente en cada cuantificación de grado. Así, podemos tener una interpretación iterativa en "Telefonea demasiado a sus amigos"; otra temporal en "Duerme poco"; otra argumental en "Has comido demasiado", y una cuarta en la que el cuantificador funciona como "ligador no selectivo" en "La gente ha leído mucho este libro" ${ }^{50}$. En nuestra opinión, aunque es evidente la presencia de un cuantificador en la estructura del predicado, ese cuantificador no es de grado, sino de nombres discontinuos y continuos, con lo que no difiere de la cuantificación sobre individuos u objetos, adoptando en estos casos la forma de cardinalidad indefinida: "Telefonea demasiado" = demasiadas veces; "Duerme poco" = pocas horas; "Has comido demasiado" = demasiada comida, y "La gente ha leído mucho este libro" = mucha gente .

El autor defiende, en cambio, una postura contundente al hablar de la denominada por él "interpretación inherente", que se da en casos como "ensuciar demasiado la ropa", "espesar más la salsa", "llenar bastante el balde" o "ensanchar más la sala", aunque también parece que en todos aquellos casos que no se ajustan a una interpretación iterativa, temporal, argumental o aquella en la que el cuantificador funciona como "ligador no selectivo", al menos por la lista que nos ofrece: "sangrar mucho", "caminar mucho", "trabajar mucho", "masticar mucho", "adentrarse mucho", "subir mucho", "preocuparse mucho", "necesitar mucho” (ibid., pp. 71-73).

Esta interpretación "inherente" tiene su origen, como señala M.A. Dalmau ${ }^{51}$-quien la ha analizado también en profundidad-en la idea de que "los items léxicos no pueden describirse mediante una mera lista de rasgos, sino que poseen una estructura interna que es de tipo composicional" y en la de que "el sig-

${ }^{50}$ I. BosQue, “Sobre la gramática de la gradación”, pp. 69-70.

51 Art. cit., p. 72. 
nificado gramatical de los predicados deriva de esa estructura (la estructura subléxica), que es la que determina su comportamiento en la sintaxis" 52 .

Volviendo al planteamiento de I. Bosque, es interesante comprobar lo que el autor declara, en el sentido de que "contra lo que se hubiera dicho seguramente desde la semántica generativa, nosotros no creemos que sea posible tener un cuantificador que modifique sintácticamente un componente de la estructura subléxica de una palabra, puesto que esa situación conculca la definición misma de sintaxis como parte de la gramática" ${ }^{53}$. Aunque esta opinión podría resultar contradictoria respecto al planteamiento general de la teoría esbozada por M.A. Dalmau, el autor español propone, no obstante, un procedimiento restrictivo mediante el cual el adjunto de grado "coteja" la presencia de un núcleo cuantificativo abstracto sobre el predicado más incrustado de la estructura léxica del verbo, con lo que conciliaría de alguna manera -si no matizaría-, este hecho con su afirmación de que "las relaciones sintácticas no pueden entrar en los componentes semánticos de las unidades léxicas"54.

Consideremos ahora de nuevo los casos, a nuestro entender desemejantes, de "Ensuciar demasiado la ropa", "Espesar más la salsa" o "Llenar bastante el balde", frente a los "Sangrar mucho", "Caminar mucho" y "Trabajar mucho", al menos. En los tres primeros casos, las expresiones correspondientes no significan que se hacen intensamente las acciones de ensuciar, espesar o llenar, ni que se alcanzan estadios elevados en ellas, sino que se llega a que la ropa esté demasiado sucia, la salsa más espesa o el balde bastante lleno. Con ello se recurre, en efecto, a un elemento de la estructura léxica, no presente en la sintaxis, sobre el que sí opera directamente el cuantificador de grado, no sobre la acción misma, en los casos que nos ocupan, los adjetivos sucio, espeso y lleno.

${ }^{52}$ El modelo propuesto se atribuye a K. Hale y S. Keyser (1993), citado por la autora, que se organiza de acuerdo con principios sintácticos tales como la Teoría de X-barra, el principio de categorías vacías, Muévase-a y la Restricción de movimiento de núcleo (cf. art. cit., p. 72).

53 "Sobre la gramática de la gradación", pp. 68-69.

54 I. BosQue, "Sobre la gramática de la gradación”, p. 68. Aunque tratamos de evitar en este trabajo ciertas manifestaciones muy técnicas de adscripción a un marco teórico determinado, reproducimos la fórmula que I. BosQUE (“Sobre la gramática de la gradación”, p. 72) utiliza para representar esta operación en uno de sus ejemplos: [SV [SV Vinergativoij [SQ [Q eij] [SN $\mathrm{Nj}]]$ muchoi $]=[$ Trabajar [trabajar [mucho trabajo] mucho]. 
Pero esta explicación invita a pensar que no estamos en estos tres casos ante una cuantificación de grado verbal, aunque sí ante una cuantificación de grado que opera sobre elementos adjetivos ("demasiado sucia", "muy espesa", "bastante lleno" ${ }^{55}$ ). Recordemos que, no obstante, este autor utiliza esta interpretación inherente también en los casos de "Sangrar bastante", "Caminar mucho" o "Trabajar mucho", donde los núcleos serían sangre, camino y trabajo; pero, a diferencia de los tres casos anteriores, en estos no creemos que estemos ante una cuantificación de grado que opera sobre adjetivos, sino ante una cuantificación de objetos continuos, con lo cual no se trataría realmente de una cuantificación de grado.

Finalmente, en los casos de "Masticar mucho", "Adentrarse mucho" y "Subir mucho", las interpretaciones de muy masticado, muy adentro y muy alto invitan a pensar nuevamente en que las auténticas variables no son los verbos sino las unidades marcadas, por cierto, unidades respecto a las que sí hemos defendido que se aplica la cuantificación de grado, y que son las que indican propiedades, $\mathrm{u}$ otras que hacen referencia a dimensiones espaciales, o temporales, con carácter 'no delimitado'. El único caso en el que consideramos que podría existir una interrelación entre grado e intensidad es en el de "Masticar mucho" = intensamente, pero se trataría, en nuestra opinión, de la confirmación de un hecho que también se da en casos como "El cielo está muy azul"56 o "Juan grita mucho", es decir, que a veces grado e intensidad se identifiquen.

\section{Conclusiones}

Básicamente, en este trabajo hemos defendido que sólo hay cuantificación de grado cuando entran en juego propiedades. Sobre

55 Se trata de adjetivos perfectivos, es decir, que indican 'estado resultante' y que presentarían un paralelismo con la característica aspectual de la 'delimitación', y, por tanto, la no cuantificación, aunque, como ha demostrado M.A. DALMAU, incluso cuando "el locatum representa una entidad material con el rasgo [-d]” (art. cit., p. 79) puede haber casos donde los verbos no son cuantificables: alambrar mucho, alfombrar mucho, asfaltar mucho, etc.

56 Véase el planteamiento de V. Demonte (art. cit., p. 174) sobre qué implica la gradación de los adjetivos de color. Podemos concluir, pues, que en estos casos de adjetivos de color la propiedad no es escalar, por lo que el cuantificador de grado informaría sobre la intensidad. 
esto hay que insistir, primero, en que el grado debe ser distinguido tanto de la noción de cantidad como de la de intensidad.

En concreto, el concepto de cuantificación de grado queda restringido, en nuestra opinión, a la idea de situar una propiedad en algún nivel dentro de una escala, y la noción que se desprende es cualitativa, o cualitativo-cuantitativa, no de intensidad; en este sentido, la escalaridad puede implicar tanto cantidad (caso de la cuantificación de individuos y objetos) como cualidad y cantidad (caso de la cuantificación de grado). No obstante, podríamos admitir también un cruce entre cualidad e intensidad ("muy azul", "masticar mucho", "gritar mucho"), lo que no quiere decir que cantidad, cualidad e intensidad deban identificarse.

Pero, además, en el caso de la llamada "cuantificación de grado verbal" estamos en realidad, como señala I. Bosque, ante una "paradoja de la gradación", ya que no se está realizando una cuantificación sobre el nivel en que tienen lugar los eventos al no existir ninguna variable verbal. $Y$ eso tanto en las interpretaciones iterativa, temporal, argumental, aquélla en la que el cuantificador actúa como ligador no selectivo, como en la llamada por este autor "interpretación inherente", aunque está claro que en todos esos casos hay un cuantificador presente en la estructura del predicado.

M. Dolores Muñoz NúÑez Universidad de Cádiz 